\title{
CYCLIC CHANGES OF BLOOD ECHOGENICITY IN HIGH-FREQUENCY ULTRASOUND
}

\author{
M. G. M. DE KroOn, ${ }^{\dagger \neq}$ C. J. SlaGer, ${ }^{\dagger}$ W. J. GuSSENhoven, ${ }^{\dagger, *}$ \\ P. W. SERruYs, ${ }^{\ddagger}$ J. R. T. C. RoElandT ${ }^{\dagger}$ and N. BOM ${ }^{\dagger}$
}

${ }^{\dagger}$ The Thoraxcenter, the University Hospital, Rotterdam, and the Erasmus University, Rotterdam, ${ }^{\ddagger}$ The TNO Institute of Applied Physics, Delft and *the Interuniversity Cardiology Institute of The Netherlands, Utrecht

\author{
(Received 22 October 1990; in final form 2 April 1991)
}

\begin{abstract}
Ultrasound images from human arteries obtained in vivo with an intravascular 30 MHz ultrasound imaging device show that blood echogenicity changes during the cardiac cycle. Quantitative measurements of blood echogenicity during the cardiac cycle suggest that these variations may be related to changes in the state of erythrocyte aggregation, which are induced by varying shear rate.
\end{abstract}

Key Words: Blood echogenicity, Red blood cell aggregation, Cardiac cycle.

\section{INTRODUCTION}

During human application of a $30 \mathrm{MHz}$ ultrasound imaging catheter device (Bom et al. 1989) the blood in the lumen shows high backscatter intensity and thus often limits the visualisation of the luminal border (Gussenhoven et al. 1990) especially when blood flow is blocked by stenosis. We feel that a better understanding of the acoustical characteristics of blood is necessary to achieve adequate signal transmission through the blood.

Spontaneous echo contrast of blood is commonly seen on standard 2D images at frequencies of 2.5-5 MHz in low cardiac output states (van Herwerden et al. 1986). Sigel et al. (1982a) showed that blood echogenicity is influenced by local shear rate and that a decreasing shear rate results in an increased echogenicity. Because erythrocytes aggregate at a decreasing shear rate and the aggregates disperse when the shear rate increases, it is assumed that the shear rate dependent state of aggregation results in changes in echogenicity (Sigel et al. 1982a, 1983; Beitler et al. 1983).

Studies so far deal only with the effect of shear rate on echogenicity of blood under stationary flow conditions. In vivo blood flow is pulsatile and thus the shear rate also changes during the cardiac cycle.

Address correspondence to: M: G. M. de Kroon, Stieltjesweg 1, 2628 CK Delft, The Netherlands.
The purpose of this study is to investigate the acoustic behavior of flowing blood in vivo during the cardiac cycle using a high frequency ultrasound (30 $\mathrm{MHz}$ ) intravascular imaging device.

\section{MATERIALS AND METHODS}

\section{Intravascular ultrasound system}

By mechanical rotation of a $30 \mathrm{MHz}$ single element transducer ( 16 rotations/s) mounted in the tip of a $5 \mathrm{~F}$ catheter, real-time cross sectional images of the arterial lumen and the vessel wall are obtained (Bom et al. 1989). During one rotation 400 pulseecho measurements are performed. Time-dependent gain is applied on each received signal to compensate for attenuation. The signals are demodulated, logarithmically amplified and digitized ( 8 bits). Finally they are transmitted to a digital scan converter and circularly displayed in video format with 256 grey levels at 50 fields/s and registered on videotape. Both the ECG and the pressure in the catheterized artery are simultaneously recorded and superimposed on the video images. In Fig. 1 a schematic diagram of the imaging device is depicted. The system gain is kept constant during the period in which data are analysed. Gain setting might be changed between subsequent catheterisations.

\section{Catheterization procedure}

Three patients who underwent diagnostic coronary angiography were asked to participate with the 


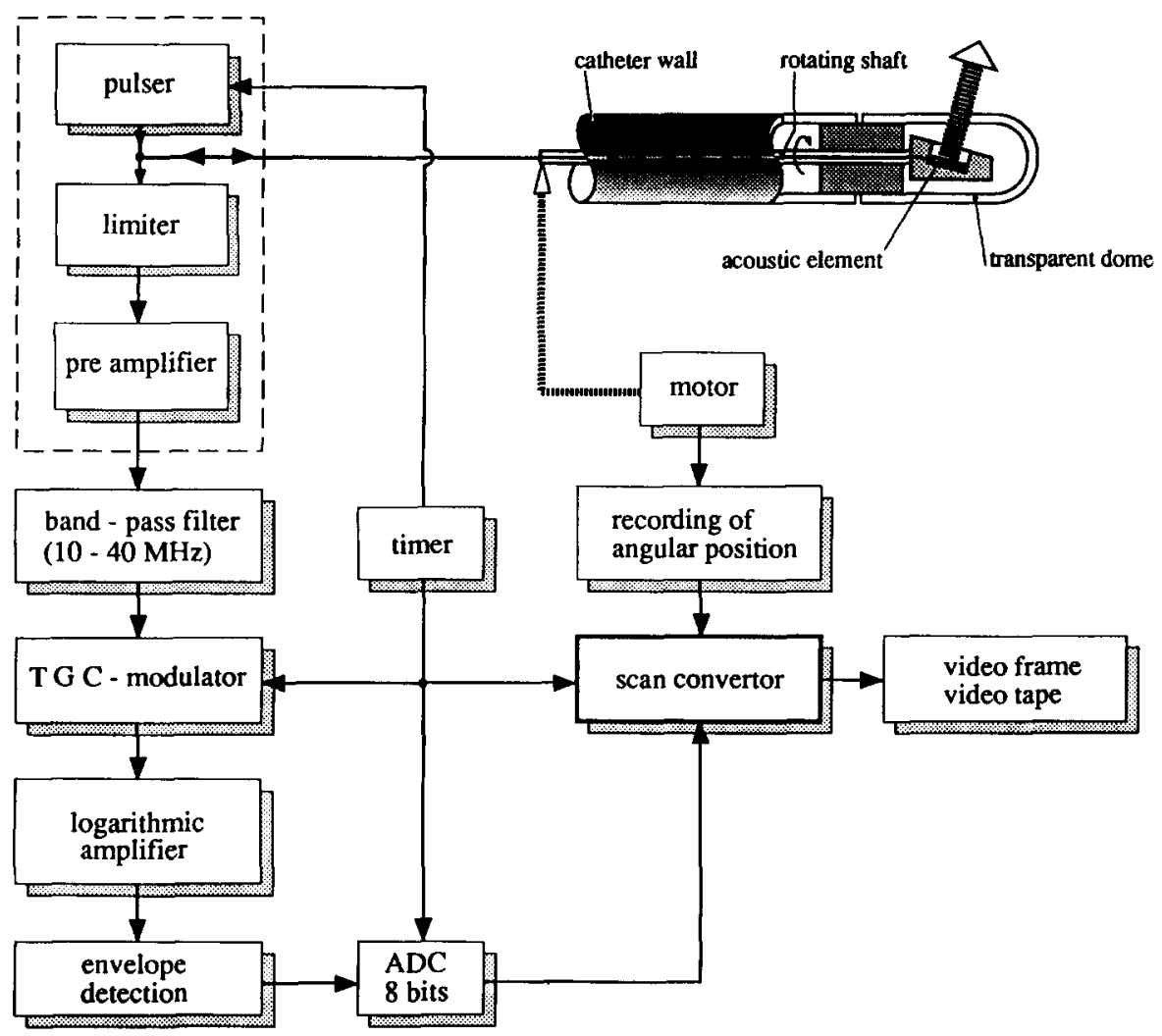

Fig. 1. Experimental set-up and data acquisition scheme of measurements of red blood cell echogenicity by an intravascular high frequency imaging device.

intravascular ultrasound study. For this purpose the femoral artery was punctured routinely and a $9 \mathrm{~F}$ sheath is introduced prior to the coronary angiography. The intravascular catheter is positioned in the iliac artery via the sheath. The evaluation of the acoustic behaviour of blood is only performed at locations where the lumen is not significantly obstructed by intimal thickening. The HTC values of the blood of the three patients varies between $38 \%-43 \%$.

\section{Qualitative evaluation}

The video images of the three patients (M.dK., C.S., N.B.) are visually evaluated by three independent investigators. The added pressure signal on the video images provides a time reference. Slowly running the tape facilitates accurate analysis.

\section{Quantitative evaluation}

A videodensitometric (ten Cate et al. 1989) system is used to quantify the blood echogenicity. This system digitizes the video signal ( 8 bits) and calculates the average grey level within a selected region in a video image. Thus the maximum video level has a value of 256.

Three regions of interest are selected in the lumen of the arterial cross section: one region close to the arterial wall (region 1), one in the center (region 2) and one near the catheter (region 3 ). The echodensity is computed in consecutive frames of a recording at a fixed catheter position. The analysis is applied to the arterial images of each patient during three cardiac cycles. The variable gain setting implies that comparison between the three patients of the absolute grey level values is not meaningful. The computed echodensity only gives information on the sign, periodicity and significance of the changes in the acoustic behaviour of blood.

\section{RESULTS}

When visually analysing the video images, a cyclic variation of blood echodensity synchronous with the cardiac cycle is observed. During diastole the echogenicity increases; and immediately after the rise of the arterial pressure (in early systole) the echoden- 


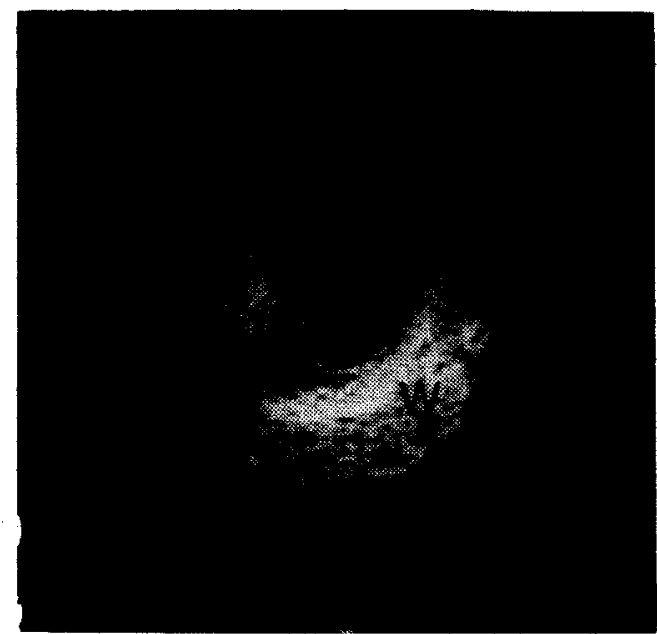

(a)

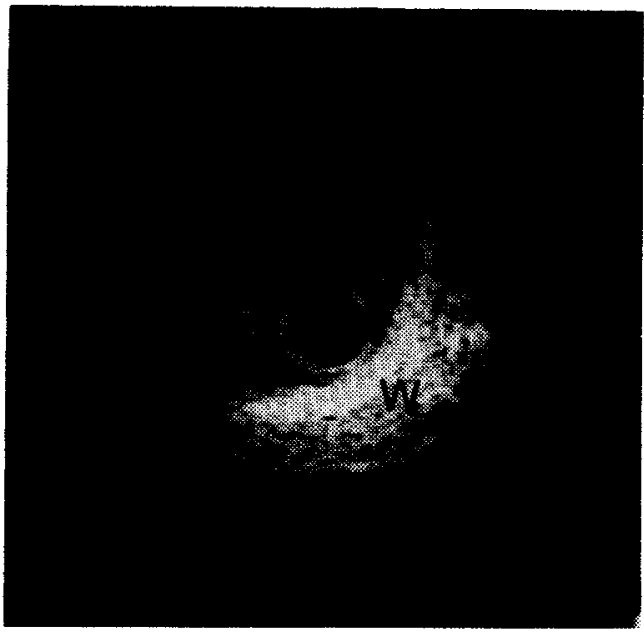

(b)

Fig. 2. B-mode picture of the iliac artery at end diastole (a) and early systole (b). L: lumen; W: arterial wall.

sity decreases. This is illustrated in Fig. 2 in which cross sectional images of an artery at end diastole and early systole of patient \#1 are shown.

In Fig. 3a the selected regions of the videodensitometric measurements of patient $\# 1$ are indicated. The calculated echodensity in these regions as a function of time and the simultaneously recorded intraarterial pressure are presented in Fig. $3 b$.

The density-versus-time plot (Fig. 3b) indicates that the variations in the blood echodensity are synchronous with the cardiac cycle: echogenicity increases during diastole and decreases rapidly at early systole. The echodensity also decreases as a function of the distance to the transducer.

In Fig. 4 the measured echodensity of all three patients at end diastole and at end systole are given.
Note that the grey level is a logarithmically scaled quantity and that absolute variations in grey level are related to relative changes in intensity.

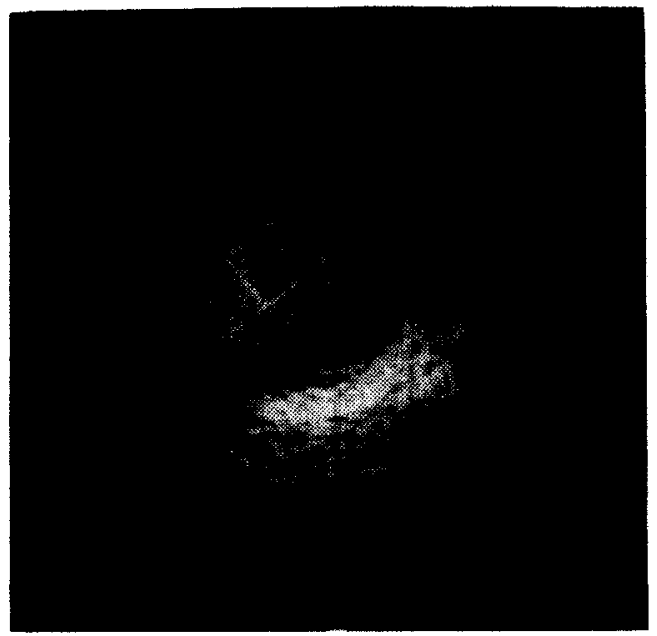

(a)

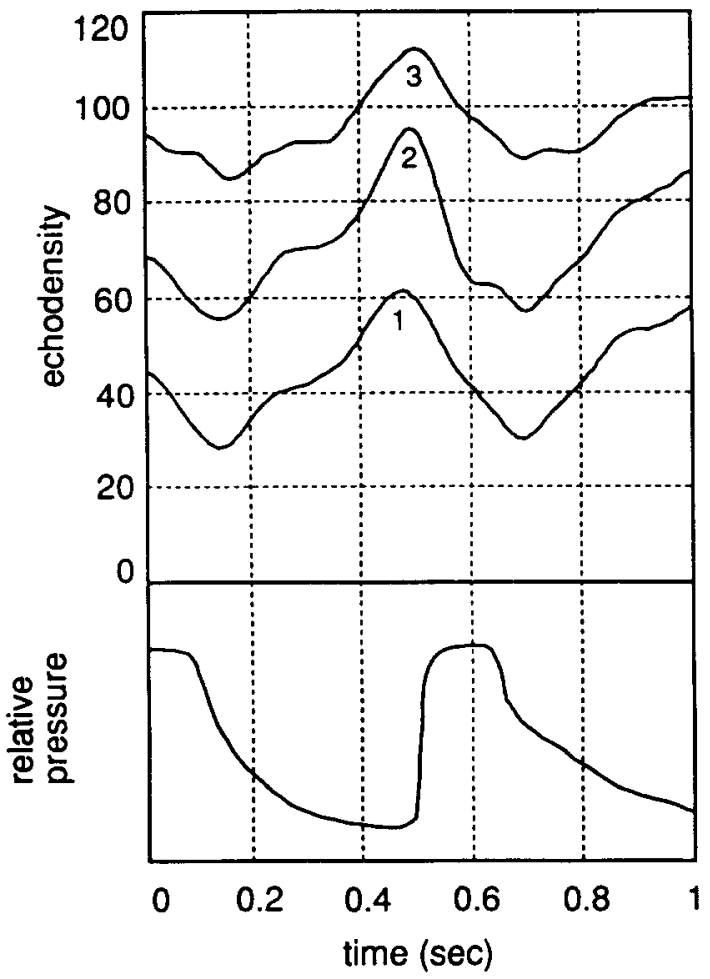

(b)

Fig. 3. (a) Selected regions in lumen of cross section of the iliac artery. Echodensity is calculated in these regions during a cardiac cycle for 50 consecutive frames. (b) Measured echodensity in the indicated regions, as a function of time, during one cardiac cycle. The blood pressure in the catheterized artery is given as a reference to the moment in the cardiac cycle. 


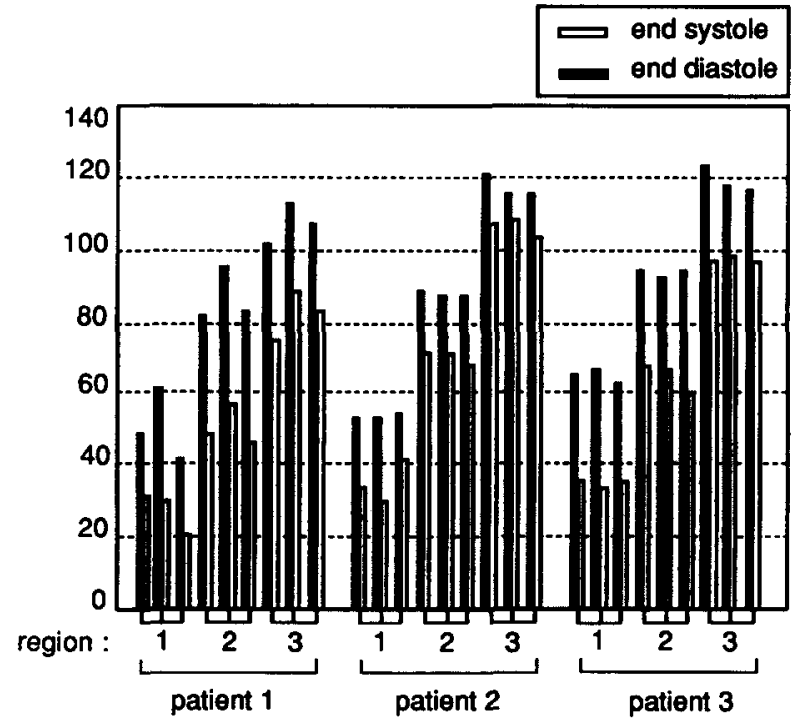

Fig. 4. Averaged echodensity in the specified regions in the lumen of three patients. Density is given at end diastole and end systole of three analyzed cardiac cycles.

\section{DISCUSSION}

The echogenicity of flowing blood may depend on several factors: concentration of red blood cells (RBCs) (Shung 1982; Shung and Lim 1990; Yuan and Shung 1988a), which may vary across the artery lumen (Goldsmith 1972; Aarts 1985; Aarts et al. 1988), deformation and alignment of RBCs and their shear rate dependent state of aggregation (Sigel 1982a, 1982b, 1983; Beitler et al. 1983) and the ultrasound frequency (Shung et al. 1976; Yuan and Shung 1988b).

According to measurements by Goldsmith (1972), Aarts (1985) and Aarts et al. (1988) the concentration of RBCs is higher in the center of the flow than near the wall, e.g., when the average HTC is $40 \%$, the local HTC level varies between $0 \%$ at the wall and $60 \%$ in the center of the flow.

Goldsmith (1971) observed that the originally disk-shaped red blood cells deform into ellipsoids and that their major axis aligns in the direction of the flow at increased shear rate.

Shung et al. (1976, 1982, 1984, 1990) and Yuan and Shung (1988a, 1988b) measured the backscatter intensity of RBCs in a Ringer's solution at varying hematocrit values and varying ultrasound frequencies (5-15 MHz). Their results show that the prepared RBC solution follows Rayleigh's scattering theory for small particles i.e., the backscatter intensity increases with the fourth power of the ultrasound frequency. They also reported that the backscatter intensity increases with increasing hematocrit up to peak values in the hematocrit range of $17 \%-24 \%$. At higher hematocrit values backscatter intensity decreases.

These measurements however were carried out under conditions that aggregation of RBCs is prevented. It is well known that under normal conditions aggregates of RBCs may be formed. The state of aggregation is affected by temperature, $\mathrm{PH}$, fibrinogen concentration and shear rate (Sigel et al. 1982b; Beitler et al. 1983). According to the studies of blood viscosity by Schmid-Schonbein et al. $(1968,1982)$ RBC aggregates disperse at an increasing velocity gradient (shear rate) and reversibly enlarge at a decreasing shear rate. Increased ultrasonic backscatter may be expected from the larger aggregates compared to the small individual RBCs $(7 \mu \mathrm{m})$. This was demonstrated by several observations showing increased echogenicity at decreasing shear rate (Sigel et al. 1982a, 1982b, 1983; Beitler et al. 1983; Yuan and Shung 1988a; Kallio and Alanen 1988).

Transit times between different states of aggregation are characterised by the half-time of aggregation. Jansonius and Bijlstra (1965) determined the halftime of aggregation by measuring the light reflection of blood (syllectometry). According to their measurements, the half-time of aggregation is approximately $10 \mathrm{~s}$ in normal blood (HTC of 40\%). Schmid-Schonbein et al. $(1975,1976)$ used a light transmission technique to determine the half-time of aggregation. They reported an aggregation half-time of $3.5 \mathrm{~s}$ of blood of normal patients. However, frequently two different half-time constants were observed: a short one in the range of $0.5-1.5 \mathrm{~s}$ and the conventional one of $3.5 \mathrm{~s}$. It was also noted that the half-time of aggregation increases strongly with decreasing erythrocyte concentration.

Although the elongation of erythrocytes and their alignment to the flow direction might have an impact on echogenicity, these features cannot explain the observed cyclic variations of the echogenicity (Goldsmith 1971; Visser 1989). Alignment of erythrocytes would enhance echogenicity during systole, which is not in accordance with the results presented here.

From the pressure curve, the arterial flow-rate curve can be estimated. The echodensity curve seems to follow the inverse of the expected flow-rate curve rather than the pressure curve. This might indicate that the echogenicity variation is a flow-related phenomenon. It is supposed that the flow-related phenomenon that causes changes in echogenicity is the shear rate dependent state of aggregation of RBCs. During diastole the decreasing blood flow velocity results in lower shear rates, enabling the formation of 
new and longer aggregates. This supposedly causes an increase in echodensity during diastole. At early systole, the higher shear rates break down the aggregates, which might result in decreasing echodensity. So far, with ultrasound observations of fast changes in the state of aggregation have not been reported. Indeed the measured half-time of aggregation (SchmidSchonbein et al. 1975, 1976) suggests that one second may be sufficient to detect changes in the state of aggregation. Moreover, applying higher frequency (30 $\mathrm{MHz})$ than in conventional ultrasonography (2.5-5 $\mathrm{MHz}$ ) allows the observation of smaller aggregates that are formed faster.

Also the shape and orientation of the aggregates may influence echogenicity. The interactive surface forces between RBCs promote a well-defined form of the aggregates, called "rouleaux." Rouleaux arise from the piling up of RBCs, which have the shape of a disk, to form a long, cylindrically-shaped aggregate (Charm and Kurland 1974). Because rouleaux disperse when they are exposed to high velocity gradients, they will align in the direction of the flow. The specific shape of rouleaux and their alignment may play an important additional role in explaining the echogenicity of flowing blood. Higher echogenicity will be observed when the direction of the sound wave is perpendicular to the alignment of the rouleaux. In the present study the direction of the sound wave is almost perpendicular to the rouleaux alignment-a good condition to measure the supposed effect of aggregation on echogenicity.

Another possible explanation of the observed variations in echogenicity is that during diastole local HTC might increase due to the low flow rate. At the beginning of systole, the accumulated RBCs might disperse and local HTC might decrease. The observed variations in echogenicity can only be explained if it is assumed that the echogenicity of the blood follows the curve as measured by Shung and Lim (1990), and that the HTC does not exceed the critical HTC value $(17 \%-24 \%)$ beyond which echogenicity decreases at increasing HTC. However, at average HTC values of almost $40 \%$, it is unlikely that the suggested echodensity variations can be explained by HTC variations. Note that the suggested HTC variations might enhance possible aggregation during diastole.

Care must be taken in mutually comparing the absolute values of the three selected regions because the echogenicity is also affected by the distance to the transducer. Attenuation, geometric spreading of the sound wave and the time dependent gain may cause distance-dependent echo variations.

Although the clinical relevance of the varying echogenicity is speculative at this moment, some potential usefulness should be discussed. Blood echogenicity may be related to the state of vascular disease. Kallio et al. (1989) observed that patients with vascular disease show higher blood echogenicity than healthy volunteers. As a consequence it may be expected that in patients with advanced vascular disease, the ultrasonic signal transmission through blood is worsened. Kallio even made the hypothetical statement that blood echogenicity might provide a more specific measure of disease than the conventional erythrocyte sedimentation rate (ESR). Application of the intravascular ultrasound imaging catheter eventually allows in vivo local study and quantitative standardisation of blood echogenicity and the supposedly related aggregation dynamics of RBCs. Local differences in echogenicity have already been observed using conventional ultrasound imaging devices (van Herwerden et al. 1986). Whether or not this observation is related to the local state of disease is a research goal. It has to be considered that the presence of a plaque may disturb normal blood flow and so may cause local changes in the state of aggregation, which may help locate the atherosclerotic plaque.

Furthermore the potential exists that local shear force conditions may be derived from the echogenicity of the flowing blood. This could open a new field of studies on the suggested relationship between local shear forces and the rate of development of arterial wall disease (Fry 1968; Caro et al. 1969).

It is concluded that the observed cyclic changes in blood echogenicity may well be explained by variations in erythrocyte aggregation as a result of changing shear rates. However, further experiments are necessary to confirm this relationship and rule out other explanations.

\section{REFERENCES}

Aarts, P. A. M. M. The role of hemodynamic factors in the interaction of blood platelets with the vessel wall. Master Thesis. The Netherlands: University of Utrecht; 1985.

Aarts, P. A. M. M.; van den Broek, S. A. T.; Prins, G. W.; Kuiken, G. D. C.; Sixma, J. J. Blood platelets are concentrated near the wall and red blood cells in the center of flowing blood. Arteriosclerosis 8(6):819-824; 1988.

Beitler, J.; Sigel, B.; Machi, J.; Justin, J. R. The effects of temperature on blood flow ultrasonic echogenicity in vitro. J. Ultrasound Med. 2:529-533; 1983.

Bom, N.; ten Hoff, H.; Lancée, C. T.; Gussenhoven, W. J.; Bosch, J. C. Early and recent intraluminal ultrasound devices. In: Bom, N.; Roelandt, J., eds. Intravascular ultrasound, techniques, developments, clinical perspectives. Dordrecht. The Netherlands: Kluwer Academic Publishers; 1989:79-87.

Caro, C. G.; Fitz-Gerald, J. M.; Schroter, R. C. Arterial wall shear distribution of early atheroma in man. Nature (London) 223:1159-1161; 1969 
ten Cate, F. J.; de Jong, N.; Mittertreiner, W.; Serruys, P. W.; Roelandt, J. R. T. C. Myocardial contrast two dimensional echocardiography. Int. J. Card. Im. 4:53-56; 1989.

Charm, S. E.; Kurland, G. S. Blood flow and microcirculation. New York: Wiley; Chapter 2 (Blood viscosity). 1974:23-63.

Fry, D. L. Acute vascular endothelial changes associated with increased blood velocity gradients. Circ. Res. 22:165-197; 1968.

Goldsmith, H. L. Deformation of human red cells in tube flow. Biorheology 7:235; 1971 .

Goldsmith, H. L. The flow of model particles and blood cells and its relation to thrombogenesis. In: Spaet, T. H., ed. Progress in hemostasis and thrombosis. New York: Grune and Stratton; 1972:97-139.

Gussenhoven, W. J.; Bom, N.; Li, W.; van Urk, H.; Pieterman, H.; van Suylen, R. J.; The, S. H. K. The hypoechoic media, a landmark for intravascular ultrasonic imaging. (Diagnostic and therapeutic cardiovascular interventions.) SPIE J 1425:203-206; 1991.

van Herwerden, L. A.; Gussenhoven, W. J.; Roelandt, J.; Bos, E.; Ligtvoet, C. M. Intraoperative epicardial two-dimensional echocardiography. European Heart Journal 7:386-395; 1986.

Jansonius, N. J.; Zijlstra, W. G. Various factors influencing rouleaux formation of erythrocytes studied with the aid of syllectometry. Proc. Kon. Ned. Akad. Wet. 68 (Zeries C):121-127; 1965.

Kallio, T; Alanen, A. A new ultrasonic technique for quantifying blood echogenicity. Investigative Radiology 23:832-835; 1988 .

Kallio, T.; Alanen, A.; Kormano, M. The in vitro echogenicity of flowing blood in patients with vascular disease and the effect of naftidrofuryl. Ultrasound Med. Biol. 15(6):555-559, 1989.

Schmid-Schonbein, H.; Gaehtgens, P.; Hirsch, H. On the shear rate dependence of red cell aggregation in vitro. J. Clin. Inv. 47:1447-1454; 1968.

Schmid-Schonbein, H.; Kline, K. A.; Heinrich, L.; Volger, E.; Fischer, T. Microrheology and light transmission of blood. III.
The velocity of red blood cell aggregation. Pflugers Arch 354:299-317; 1975.

Schmid-Schonbein, H.; Gallasch, G.; von Gosen, J.; Volger, E.; Klose, H. J. Red cell aggregation in blood flow. I. New methods of quantification. Klin. Wschr. 54:149-157; 1976.

Schmid-Schonbein, H.; Volger, E.; Kiesewetter, H.; Dauer, U.; Heilmann, L. New hemorheological techniques for routine laboratory. Clinical Hemorheology 2:99-105; 1982.

Shung, K. K.; Sigelmann, R. A.; Reid, J. M. Scattering of ultrasound by blood. IEEE Trans. Biomed. Eng. 23(6):460-467; 1976.

Shung, K. K. On the ultrasound scattering from blood as a function of hematocrit. IEEE Trans. Son. Ultrason. 29(6):327-331; 1982.

Shung, K. K.; Yuan, Y. W.; Fei, D. Y. Effect of flow disturbance on ultrasonic backscatter from blood. J. Acous. Soc. Am. 75(4): $1265-1272 ; 1984$.

Shung, K. K.; Lim, C. The effect of hematocrit on Doppler spectrum. J. Ultrasound Med. 9:S51:Abstract \#1305; 1990.

Sigel, B.; Machi, J.; Beitler, J. C.; Justin, J. R.; Coelho, J. C. U. Variable ultrasound echogenicity in flowing blood. Science 218:1321-1323; 1982a.

Sigel, B.; Coelho, J. C. U.; Schade, S. G.; Justin, J.; Spigos, D. G. Effect of plasma proteins and temperature on echogenicity of blood. Investigative Radiology 17:29-33; 1982b.

Sigel, B.; Machi, J.; Beitler, J. C.; Justin, J. R. Red cell aggregation as a cause of blood flow echogenicity. Radiology 148:799-802; 1983.

Visser, K. R. Electric properties of flowing blood and impedance cardiology. Annals of Biomed. Eng. 17:463-473; 1989.

Yuan, Y. W.; Shung, K. K. Ultrasonic backscatter from flowing whole blood. I: Dependence on shear rate and hematocrit. J. Acous. Soc. Am. 84(1):52-58; 1988a.

Yuan, Y. W.; Shung, K. K. Ultrasonic backscatter from flowing whole blood. II: Dependence on frequency and fibrinogen concentration. J. Acous. Soc. Am. 84(4):1195-1200; 1988b. 